\title{
The effect of the COVID-19 lockdown on children with asthma-related symptoms: A tertiary care center experience
}

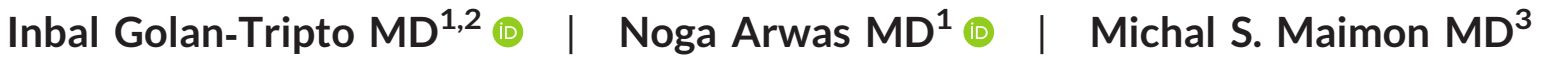

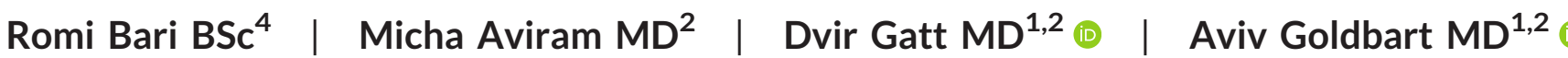

${ }^{1}$ Department of Pediatrics, Soroka University Medical Center, Faculty of Health Sciences, Ben-Gurion University of the Negev, BeerSheva, Israel

${ }^{2}$ Pediatric Pulmonary Division, Soroka University Medical Center, Faculty of Health Sciences, Ben-Gurion University of the Negev, Beer-Sheva, Israel

${ }^{3}$ Department of Pediatric Emergency Medicine, Faculty of Health Sciences, BenGurion University of the Negev, Beer-Sheva, Israel

${ }^{4}$ Clinical Research Division, Soroka University Medical Center, Faculty of Health Sciences, Ben-Gurion University of the Negev, BeerSheva, Israel

\section{Correspondence}

Inbal Golan-Tripto, MD, Pediatric Pulmonary Division, Soroka University Medical Center, Faculty of Health Sciences, Ben-Gurion University of the Negev, PO Box 151, Beer Sheva 8410101, Israel.

Email: Inbalgt@Clalit.org.il

\begin{abstract}
Background: Acute asthma exacerbations are a common cause for emergency department (ED) visits and hospitalizations in children. Since the outbreak of coronavirus disease 2019 (COVID-19) and the education system closure/total lockdown in Israel on March 2020, we have noticed a decrease in pediatric ED visits and an increase in hospitalizations of asthma exacerbations.

Objective: to examine the patterns of ED visits for asthma exacerbations during COVID-19 outbreak, in comparison to the previous year.

Methods: A retrospective study comparing asthma related ED visits and hospitalizations among children aged 2-18 years at a tertiary center in southern Israel. Three time periods were selected: $2020 \mathrm{~A}$ (prelockdown, $2 / 1 / 20$ to $3 / 14 / 20$ ), 2020 B (lockdown, 3/15/20 to 5/15/20) and 2020 C (postlockdown, 5/16/20 to 6/30/20) and compared to the three parallel time periods in 2019. Data regarding demographics, number of ED visits and clinical severity parameters were collected and analyzed.
\end{abstract}

Results: Five hundred and twelve children visited the ED for asthma exacerbation: 273 children during 2019 and 239 children during 2020. Lockdown period in 2020 revealed significantly fewer ED visits per day compared to the parallel calendar period in 2019 (1.8 vs. 1.43, $p<.001)$. Significantly higher hospitalization rate ( $47.1 \%$ vs. $33.7 \%, p=.05)$ and longer length of stay (3.15 vs. 1.9 days, $p=.03$ ) were observed during the lockdown.

Conclusion: Lockdown is associated with fewer ED visits for asthma exacerbation, probably due to; reduced exposure to viral infections and environmental allergens, decreased availability of primary physicians and families' reluctance to arrive to the ED. ED visits during lockdown were characterized by higher hospitalization rate and longer LOS.

\section{KEYWORDS}

asthma, children, COVID-19, emergency department, lockdown 


\section{1 | INTRODUCTION}

The coronavirus disease 2019 (COVID-19) outbreak has led, since the beginning of 2020 , to the implementation of "social distancing" practices including school closures in many countries, to reduce the transmission of the disease. ${ }^{1}$ When we analyze the "lockdown approach" during influenza pandemic, a mean reduction of $29.6 \%$ in the peak of the epidemic after school closure was reported by a systematic review of 31 studies. $^{2}$ Another review presented a substantial reduction of up to $50 \%$ in the transmission of disease among children after school closure during influenza outbreak. ${ }^{3,4}$

To date, children appear to represent a low proportion out of total confirmed COVID-19 cases and are usually asymptomatic or present with mild symptoms. ${ }^{5}$

Asthma is the most common pediatric chronic respiratory disease. Acute exacerbations continue to be a major health concern among children worldwide and a common reason for emergency department (ED) visits and hospitalizations in the pediatric age. In a study examining asthma-related ED visits and hospital admissions in the United State between 2010 and 2015, acute asthma exacerbations accounted for $3 \%$ of ED visits and $6 \%$ of hospital admissions among children aged 5-17 years. ${ }^{6}$ Respiratory tract infections and allergen exposure have been recognized as the most common triggers for asthma exacerbations in children, with suggested synergistic interactions between these factors. ${ }^{7}$ The seasonal pattern of asthma exacerbations is well established. ${ }^{8}$ A typical peak is in September, when children return to school after the summer break. In schoolaged children, another peak has been viewed during the spring months, that can be attributed to the spread of pollen allergens, also known as a trigger for asthma exacerbation in this age group. ${ }^{9}$ There is scarcely any data on whether childhood asthma constitutes a risk factor for COVID-19 severity. ${ }^{10}$

Recent data suggests blood eosinophil counts may have a prognostic value in COVID-19, with eosinopenia indicating a severe disease. $^{11,12}$

Since the introduction of the COVID-19 outbreak and education system closure in Israel on March 15, 2020, we have noticed a decrease in pediatric ED visits, specifically for asthma related visits, comparing to previous years. Similar observations were recently reported with a decline of $76 \%$ in the asthma related ED visits, and a lower hospitalization rate, during COVID-19 lockdown. ${ }^{13}$

Therefore, we aim to examine the patterns of pediatric ED visits for asthma exacerbations during the COVID-19 outbreak, prelockdown, during lockdown and post lockdown, in comparison to the previous year.

\section{2 | METHODS}

This is a descriptive, cross-sectional study, comparing the asthma related ED visits and hospital admissions in Soroka University Medical Center, a single tertiary center in southern Israel, among children aged 2-18 years. Three time periods were selected: $2020 \mathrm{~A}$ (pre-lockdown, 2/1/20 to 3/14/20), 2020B (lockdown, 3/15/20 to 5/ $15 / 20$ ) and $2020 \mathrm{C}$ (post- lockdown, 5/16/20 to 6/30/20) and were compared to three parallel time periods in 2019.

\section{1 | Patients}

Pediatric patients aged 2-18 years that presented to the ED during one of the selected time periods for asthma related symptoms (e.g., shortness of breath, wheezing, tachypnea), and were treated during the visit with anti-asthmatic medications, were included in the study. Using electronic health record data, we collected the parameters for each patient, including relevant medical history and medications. Data collected included: demographic data, chronic diagnoses, medications prescribed, vital signs on admission, radiological findings, treatment at the ED, triage acuity during presentation according to Canadian Triage assessment score, ${ }^{14}$ pediatric ward or pediatric intensive care unit (PICU) admissions and length of hospitalization stay (LOS). The study received the approval of the institutional ethics committee (No. 184-20).

Our primary outcome was number of visits to the ED for asthma related symptoms during 2020 A, 2020 B and 2020 C, in comparison to 2019 A, 2019 B and 2019 C. Our secondary outcomes were clinical severity parameters during the visits, indicated by vital signs, radiology findings, rate of hospitalizations, rate of hospitalizations in PICU, LOS and LOS in the PICU. We hypothesized significantly fewer visits during lockdown, with higher severity parameters. We expected increase in ED visits after the lockdown, similar to the "September pandemic," after summer vacation. ${ }^{8}$

\subsection{Statistical analysis}

Comparisons of demographic, clinical and chest X-ray imaging as well as outcomes of admissions were performed with appropriate univariate analyses. Specifically, nominal variables were compared using Pearson's $\chi^{2}$ test, continuous variables that matched parametric criteria were compared by using Student's $t$ test or one-way analysis of variance, and ordinal variables and continuous variables that did not match parametric criteria were compared by using Wilcoxon or Mann-Whitney $U$ tests. Continuous variables are presented as mean $\pm S D$. Categorical data are expressed as percentages. Statistical significance was defined as $p \leq .05$. Analyses were performed via IBM SPSS software version 22.

\section{3 | RESULTS}

A total of 512 children presented to the ED for asthma related symptoms during the study periods: 273 children during 2019 (124 in 2019 A, 89 in 2019 B and 60 in 2019 C) and 239 children during 2020 (125 in 2020 A, 51 in 2020 B and 63 in 2020 C). Demographic data were similar except for their age, which was younger during 


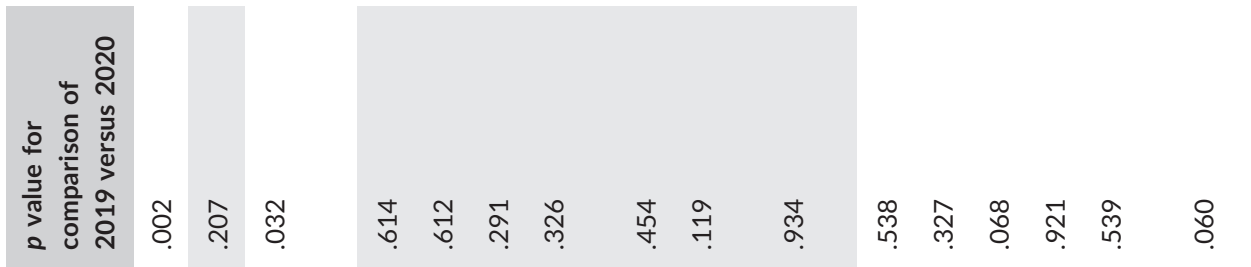

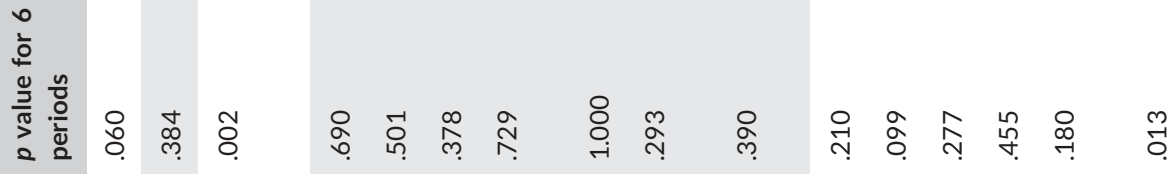

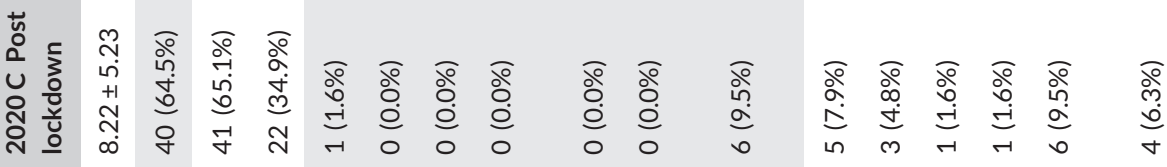

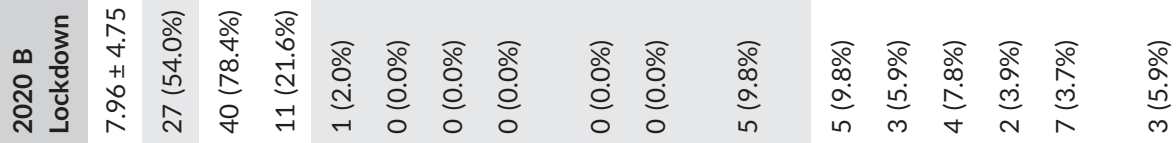

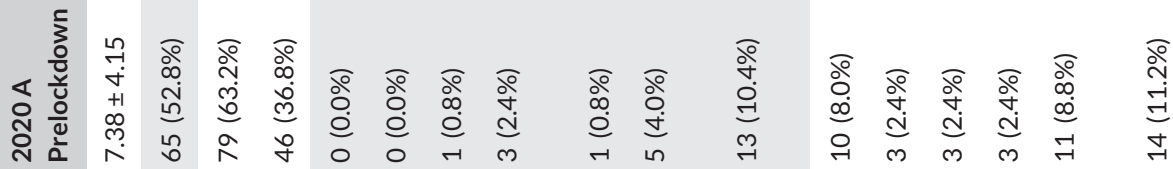

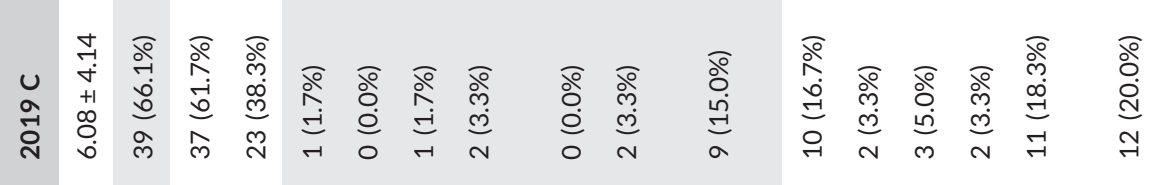

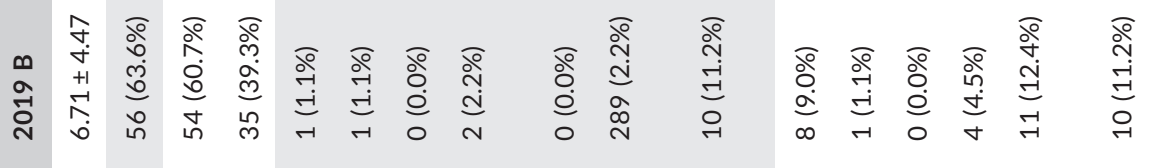

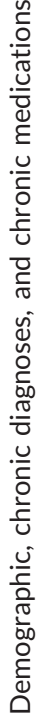

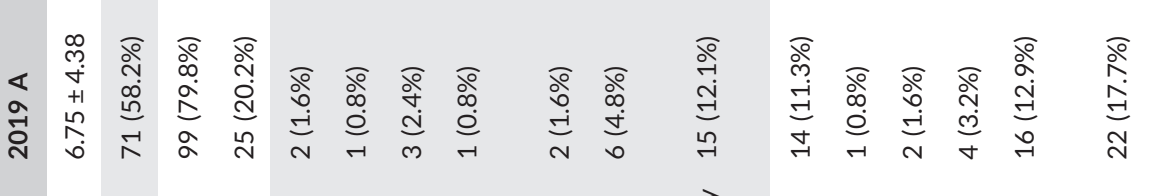

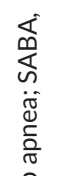

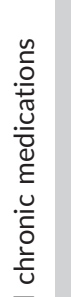

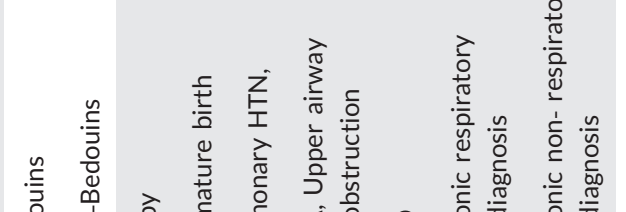


2019; median age of 5.2, 5.3, and 4.7 years during 2019 A, 2019 B and 2019 C compared with 6.7, 6.7, and 6.6 years during 2020 A, $2020 \mathrm{~B}$, and $2020 \mathrm{C}$, respectively $(p=.06)$. Male and Bedouin Arab descent were the majority of the study population in all periods. The proportion of visits by Bedouin patients during lockdown, was higher compared with the parallel period in 2019 (60.7\% in 2019 B vs. $78.4 \%$ in $2020 \mathrm{~B}, p=.002$ ). Similar number of patients with chronic non-respiratory diseases (e.g., epilepsy, Down syndrome) and chronic respiratory diseases (bronchopulmonary dysplasia, pulmonary hypertension and obstructive sleep apnea) or atopy was observed during six time periods. During 2020 C period, eleven children were tested for COVID-19, using polymerase chain reaction (PCR) in nasal swab, with one positive test; a 16 years old girl with dry cough, no fever or desaturation, that was discharge from the ED. Data regarding the use of chronic respiratory medications; inhaled corticosteroids, long acting beta agonist, short acting beta agonists, and leukotriene receptor antagonist is presented in Table 1.

We identified a decrease in use of chronic respiratory treatment in 2020 compared with 2019; 17.7\%, 11.2\% and 20\% during 2019 A, $2019 \mathrm{~B}$, and $2019 \mathrm{C}$ in comparison to $11.2 \%, 5.9 \%$, and $6.3 \%$ during 2020 A, 2020 B and 2020 C respectively $(p=.013)$. Mean vital signs (fever, blood pressure and oxygen saturation on room air) were similar between time periods, except for heart rate (124 vs. 115 beats per minute, $p=.039$ ) and respiratory rate (30 vs. 25 breaths per minute, $p=.005$ ) that were lower during 2020, compared with 2019. Similar rate of abnormal radiological findings included bilateral hyperinflation, lobar infiltrates and atelectasis was observed (Table 2). Treatment during ED visit including oxygen, inhaled beta agonist (with or without inhalation of sodium chloride $[\mathrm{NaCl} 0.9 \%]$ ) and anticholinergic agents, systemic steroids, inhaled steroids, antihistamines and antibiotics (Table 3). Treatments were similar between periods, except for sodium chloride inhalation, which significantly decreased during 2020 compared with 2019; 48.4\%, 48.3\%, and 58.3\% during 2019 A, 2019 B, and 2019 C in comparison to 52\%, 19.6\%, and 22.2\% during 2020 A, 2020 $\mathrm{B}$, and $2020 \mathrm{C}$ respectively ( $p=.002)$.

There was no difference in the triage acuity evaluation on admission to the ED. We report a significantly fewer ED visits during the lockdown period, comparing to the parallel period in 2019. A clear trend was demonstrated during 2019 with fewer visits, as time goes by, in the spring and summer time. During 2020 we demonstrated similar visits' numbers before lockdown (124 vs. 125 visits), with significant decrement during lockdown (89 vs. 51 visits) and increment post lockdown (60 vs. 63 visits). When standardized for number of visits per day, as the duration of each time period was different (A: 6 weeks, B: 8 weeks, and C: 6 weeks), similar results were observed. Number of ED visits per day was 3.3, 1.8 and 1.7 during 2019 A, 2019 B, and 2019 C respectively, in comparison to 3.1, 1.4, and 2 during 2020 A, 2020 B, and 2020 C respectively $(p<.001)$, Figure $1 \mathrm{~A}$. The significant decrease in ED visits between 2019 B and 2020 B, was attributed mainly to the decrease in visits number of non-Bedouin children; 35 visits in 2019 B compared to only 11 visits in 2020 B, while in Bedouin children; 54 visits in 2019 B compared to 40 visits in 2020 B (Table 3).
The number of visits per day increased significantly in the era after lockdown when comparing 2020 C to 2020 B (2 vs. 1.43 visits per day, $p<.001)$ as well as when compared to the same time period in 2019 ( 2 vs. 1.76 visits per day $(p<.001)$ (Table 3$)$.

During lockdown, hospitalization rate was higher (47.1\% vs. $33.7 \%$, $p=.05$ ) as was length of stay (3.15 vs. 1.9 days, $p=.03$ ), possibly indicating higher severity (Figure 1B). Only six patients were admitted or transferred to the PICU during six time periods, four of them necessitated high flow nasal cannula ventilation. There was no difference in PICU admissions rate or LOS in PICU, between time periods.

\section{4 | DISCUSSION}

In this descriptive cross-sectional study, including 512 children who presented to the ED for asthma related symptoms during three time periods in two consecutive years, we demonstrated different patterns in ED visits and in hospitalizations during pre-lockdown, lockdown and post lockdown periods, compared with 2019.

There was no difference in the demographic data between the groups except for trend towards older age during 2020 compared with 2019. Male predominance, as can be expected among this prepubertal age group, was observed. ${ }^{15}$ The majority of our patients during all time periods were of Bedouin Arab descent, with a significant increase in their visits during lockdown. This could be explained by the low socioeconomic status of the Bedouin population in southern Israel, some which are living in large families with overcrowding, poor accommodations/housing conditions, and limited access to health care in some of the settlements. ${ }^{16}$ In those areas, social distancing is more difficult to achieve and the exposure to outdoor allergens was probably not significantly reduced, even during the lockdown. The mildly lower heart rate and respiratory rate noticed in 2020 is clinically insignificant and could stem from the difference in patients age between 2019 and 2020, with an average of 1.5 years older in 2020. Since asthma severity parameters (hospitalization rate and LOS) were higher during 2020 B, we can assume that this change in vital signs did not reflect milder cases, but probably proportionate to an older age. Treatment in the ED was similar between all time periods except for a significant drop in the use of NACL $0.9 \%$ inhalations during 2020 B and 2020 C. Since the COVID-19 outbreak, in accordance with the Israeli pediatric pulmonology society guidelines, there has been a shift from using smallvolume nebulizers to metered-dose inhalers with valved-holding chambers for beta-agonist and anticholinergic inhalations to reduce the viral infection transmission by reducing the spread of aerosol mass by small-volume nebulizers. ${ }^{17,18}$

The accumulating evidence on the impact of COVID-19 pandemic on ED visits patterns, show a substantial decrease in the volume of patients presenting to the ED during the pandemic, throughout various disciplines and countries. ${ }^{19}$ Furthermore, there are increasing reports on higher hospital admission rates, indicating higher acuity patients, with increased morbidity and mortality due to delayed medical care in non-COVID-19 emergency conditions. ${ }^{20-22}$ 
TABLE 2 Vital signs, and radiological findings at the ED

\begin{tabular}{|c|c|c|c|c|c|c|c|c|}
\hline ED measurements & 2019 A & 2019 B & 2019 C & $\begin{array}{l}2020 \text { A Pre- } \\
\text { lockdown }\end{array}$ & 2020 B Lockdown & $\begin{array}{l}2020 \text { C Post- } \\
\text { lockdown }\end{array}$ & $\begin{array}{l}p \text { value } \\
\text { for } 6 \\
\text { periods }\end{array}$ & $\begin{array}{l}p \text { value for } \\
\text { comparison } \\
\text { of } 2019 \\
\text { versus } 2020\end{array}$ \\
\hline ED Urgency & 3 & 3.5 & 3 & 3 & 4 & 3 & .231 & .072 \\
\hline Median & 1.5 & 1.5 & 1.5 & 1.5 & 1.5 & 1.5 & & \\
\hline BP systolic & $110.31 \pm 12.44$ & $110.93 \pm 10.72$ & $106.77 \pm 12.71$ & $110.76 \pm 12.31$ & $108.47 \pm 14.34$ & $112.18 \pm 13.87$ & .298 & .518 \\
\hline Mean $\pm S D$ & 110.43 & 109.0 & 108.0 & 110.0 & 108.0 & 112.0 & & \\
\hline \multicolumn{9}{|l|}{ Median } \\
\hline BP diastolic & $67.35 \pm 11.75$ & $66.69 \pm 10.32$ & $65.85 \pm 10.11$ & $68.04 \pm 11.82$ & $65.95 \pm 9.87$ & $68.98 \pm 11.14$ & .663 & .363 \\
\hline Mean $\pm S D$ & 129.0 & 129.0 & 126.0 & 128.0 & 118.5 & 115.0 & & \\
\hline \multicolumn{9}{|l|}{ Median } \\
\hline $\mathrm{RR}$ & $34.18 \pm 12.05$ & $33.13 \pm 12.59$ & $35.69 \pm 12.58$ & $32.10 \pm 15.03$ & $29.00 \pm 11.13$ & $31.19 \pm 13.26$ & .005 & $<.001$ \\
\hline Mean $\pm S D$ & 32.04 & 32.0 & 30.0 & 28.0 & 26.0 & 26.0 & & \\
\hline \multicolumn{9}{|l|}{ Median } \\
\hline SAT & $95.98 \pm 4.86$ & $96.73 \pm 3.33$ & $95.22 \pm 5.68$ & $96.07 \pm 3.85$ & $96.86 \pm 3.98$ & $97.27 \pm 3.49$ & .077 & .201 \\
\hline Mean $\pm S D$ & 97.00 & 98.00 & 97.00 & 97.00 & 98.00 & 99.00 & & \\
\hline Temperature & $37.71 \pm 1.11$ & $37.54 \pm 0.92$ & $37.28 \pm 0.75$ & $37.59 \pm 0.90$ & $36.64 \pm 1.08$ & $37.32 \pm 0.77$ & .100 & .959 \\
\hline Mean $\pm S D$ & 37.00 & 37.00 & 37.00 & 37.00 & 37.00 & 37.00 & & \\
\hline \multicolumn{9}{|l|}{ Median } \\
\hline $\begin{array}{l}\text { Hyperinflation } \\
\text { chest X-ray, } \\
n(\%)\end{array}$ & 26 (21.0\%) & 14 (15.7\%) & $14(23.3 \%)$ & 23 (18.4\%) & 7 (13.7\%) & 12 (19.0\%) & .523 & .758 \\
\hline $\begin{array}{l}\text { Atelectasis/ } \\
\text { filtration, } \\
n(\%)\end{array}$ & 11 (8.9\%) & 12 (13.5\%) & 10 (16.7\%) & 12 (9.6\%) & $6(11.8 \%)$ & 3 (4.8\%) & .255 & .306 \\
\hline
\end{tabular}

Abbreviations: BP, blood pressure; ED, emergency department; HR, heart rate; RR, respiratory rate; SAT, oxygen saturation.

In this descriptive cross-sectional study, we compare three parallel time periods during two consecutive years, and show a significant decrease in pediatric asthma related ED visits during COVID-19 lockdown. Similar trends in asthma related ED visits were observed in different countries, and possible explanations were suggested by the different authors. ${ }^{13,23-26}$ Since the major triggers for asthma exacerbation in the pediatric population are viral infections, it was expected that social distancing measures during lockdown will decrease viral infections transmission, including Influenza virus, Rhinovirus and Respiratory syncytial virus. ${ }^{27,28} \mathrm{An}$ other important trigger is outdoor exposure to pollen allergens and air pollution, ${ }^{29}$ again substantially reduced due to minimal exposure during lockdown and the reduction of industrial work. ${ }^{30}$ Reduced physical activity during lockdown was described in large 
TABLE 3 Treatments administered at the ED and clinical severity outcomes in 2019 and 2020

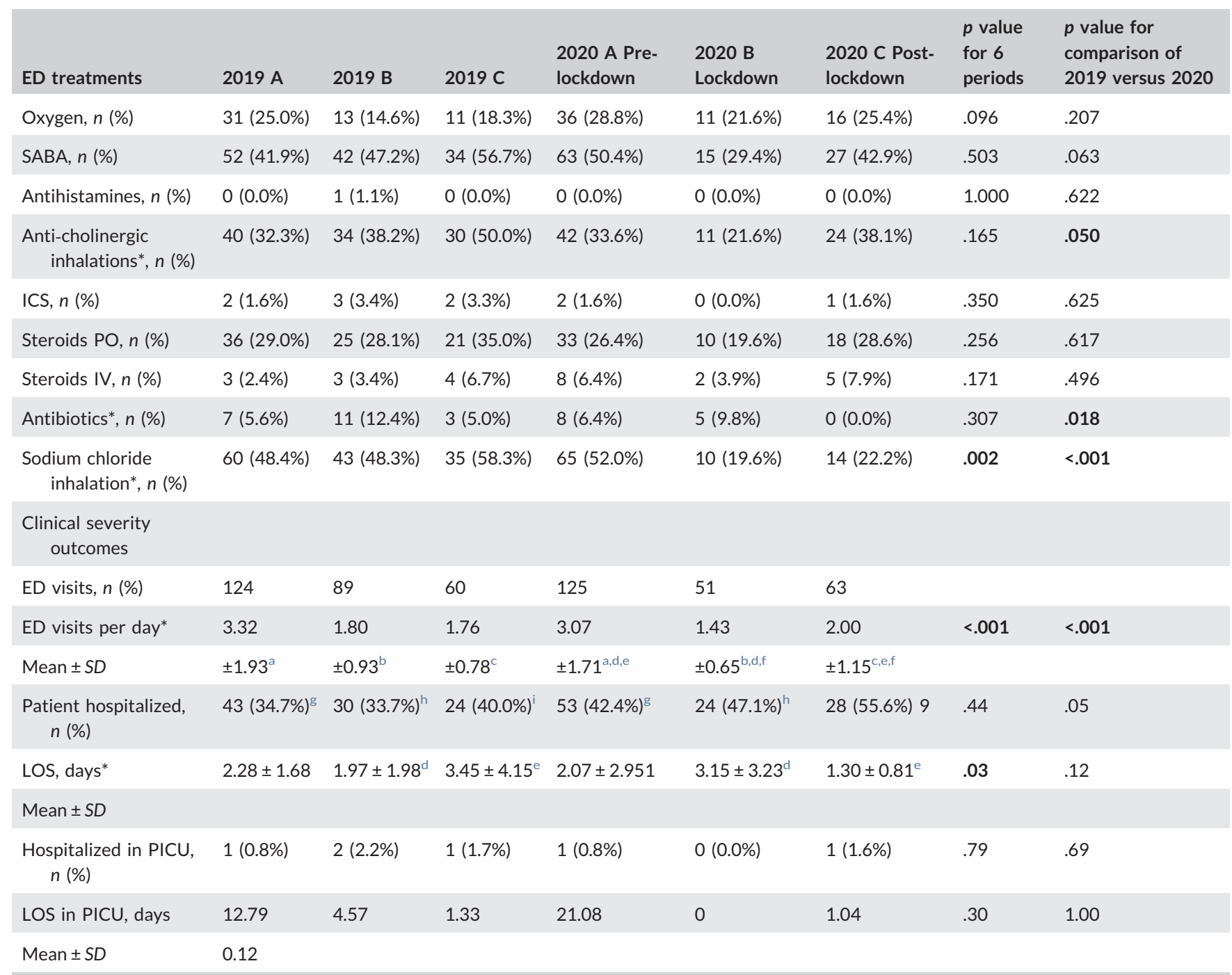

Note: ${ }^{*}$ Represent parameters with $p<.05$. Bold $p$ values also represent $p<.05$.

Abbreviations: ED, emergency department; ICS, inhaled corticosteroids; LOS, length of stay; NS, not significant; PICU, pediatric intensive care unit; PO, per os; SABA, short acting beta agonist.

${ }^{a}$ Comparison between 2019 A and 2020 A periods, $p$ value NS.

${ }^{\mathrm{b} C}$ Comparison between $2019 \mathrm{~B}$ and 2020 B periods, $p$ value NS.

${ }^{\mathrm{c} C o m p a r i s o n}$ between $2019 \mathrm{C}$ and $2020 \mathrm{C}$ periods, $p$ value NS.

${ }^{\mathrm{d} C}$ Comparison between $2020 \mathrm{~A}$ and $2020 \mathrm{~B}$ periods, $p<.001$.

${ }^{e}$ Comparison between $2020 \mathrm{~A}$ and $2020 \mathrm{C}$ periods, $p<.01$.

${ }^{f}$ Comparison between $2020 \mathrm{~B}$ and $2020 \mathrm{C}$ periods, $p$ value NS. Patients hospitalization.

${ }^{g}$ Comparison between 2019 A and 2020 A periods, $p$ value NS.

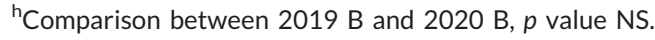

'Comparison between 2019 C and 2020 C periods, $p$ value NS. Length of stay.

questionnaires studies, along with an increase in children's psychological and behavioral symptoms and elevated screen-time. ${ }^{31-33}$ Socioaffective complications and insufficient physical activity were underscored as two of the main concerns, particularly among socioeconomic deprived children. ${ }^{34}$ As a result, a reduction in physical activities reduces the number of asthma exacerbations related to exercise induced bronchospasm and exercise induced asthma. The fewer ED visits during lockdown can also result from better asthma control while the parents are at home, providing better adherence to anti asthmatic controllers therapy. ${ }^{35}$ Although, this issue is most probably of limited effect, since most of the parents that participated in a telephone visit during lockdown, stated they stopped the preventive therapy as their child was feeling well. Another issue that should be discussed is the avoidance of approaching for medical care 
(A)

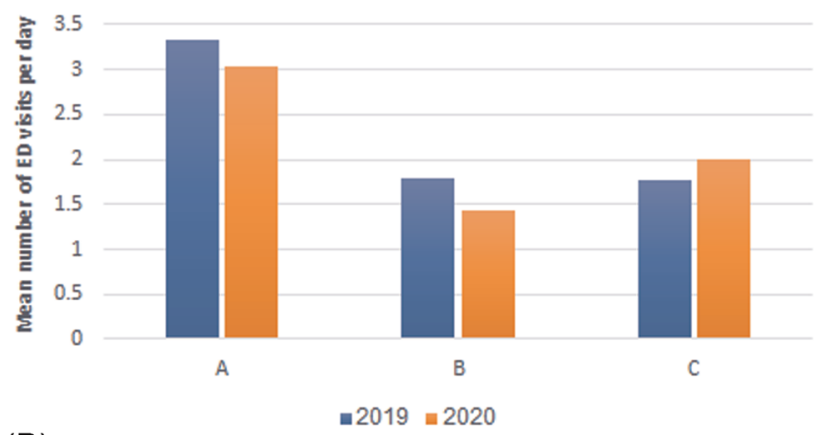

(B)

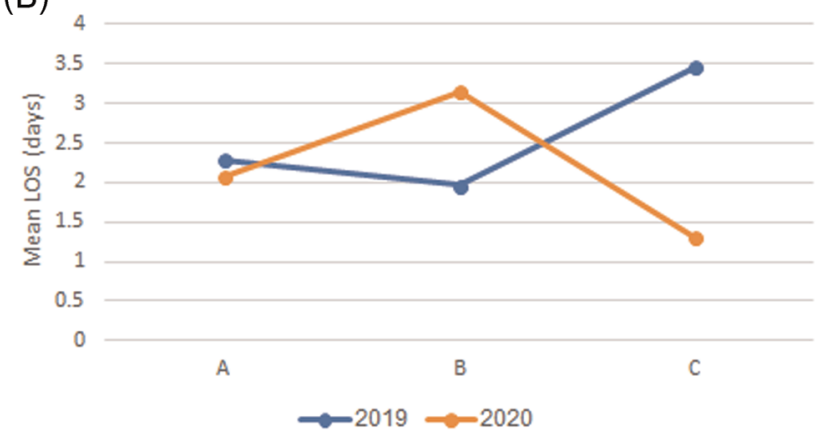

FIGURE 1 (A) ED visits per day in 2019 and 2020. (B) Mean LOS in 2019 and $2020.2 / 1 / 19$ to $3 / 14 / 19$ in 2019 and $2 / 1 / 20$ to $3 / 14 / 20$ in 2020 (pre-lockdown) $3 / 15 / 19$ to $5 / 15 / 20$ in 2019 and $3 / 15 / 20$ to $5 / 15 / 20$ in 2020 (lockdown) 5/16/19 to $30 / 6 / 19$ in 2019 and 5/16/20 to 6/30/20 in 2020 (postlockdown) ED, emergency department; LOS, length of stay [Color figure can be viewed at wileyonlinelibrary.com]

during lockdown, due to concerns of parents from increased exposure and the risk of COVID-19 transmission.

After lockdown, we demonstrated significant rise in number of ED visits, even in comparison to same time period during the previous year. In Israel, children younger than 12 years of age returned back to school after lockdown, while adolescences (aged 12-18 years) attended home school program. When we looked at the subgroup of children aged 12-18 years, the visit's numbers were similar between different study periods. That fact actually correlates with our hypothesis that everyday exposure to classmates is one of the major triggers for asthma exacerbation, and because this age group was in home schooling program during 2020 C, it was not affected by lockdown opening. We speculate that post lockdown rise represents the return to routine activity with everyday exposure to classmates, outdoor pollen exposure, rise in air pollution and physical activity, especially in the younger sub-group, aged 2-12 years. This may resemble "September pandemic," when children returning to school after summer vacations with significant rise in asthma exacerbations. $^{8}$

Alongside with a significant reduction in ED visits for asthma exacerbations during lockdown, higher hospitalization rates and longer LOS were observed, that may indicate more severe exacerbations predominate this period. This observation could be attributed to delay in presentation both due to reduced availability of community medical services during lockdown and transition towards telemedicine-based practice as well as the hesitation to come to the ED due to the fear to contract COVID-19. Our data indicated that hospitalization rate was 1.5 times higher during lockdown period compared to the previous year, in contrary to other publications that reported a reduction in the number of hospitalizations in the pediatric population during this period. ${ }^{25,36}$ We believe this fact is related to different demographics between countries, with majority of Bedouin Arab population in our region. Another possible explanation for the higher admission rate and longer LOS seen during lockdown could be a lower threshold for admission at the ER and higher threshold for discharging the patient from the wards, in light of the reduced availability of community health care services and the concern of lack of proper follow-up in the community.

Our study has a few limitations. This is a single tertiary center experience, and therefore can be influenced, as mentioned, by the unique demographics in the region. Another limitation is the retrospective nature of our study, with all data drawn from electronic files. We did not capture data regarding viral PCR in nasal swabs, which could emphasize the role of viral infections as a trigger for exacerbation during different study periods. Some of the data that was documented in a hard copy, in extremely acute patients treated in the resuscitation room, may be missing in the electronic files (e.g., intravenous Magnesium Sulfate).

In conclusion, we report a new pattern of ED visits and hospital stay of children with asthma related symptoms, associated with the COVID-19 pandemic, that perhaps is not only confined to asthma. Pediatricians should be aware to this phenomenon at the community and hospital levels.

\section{CONFLICT OF INTERESTS}

The authors declare that there are no conflict of interests.

\section{AUTHOR CONTRIBUTIONS}

Inbal Golan-Tripto completed conceptualization (lead); investigation (lead); methodology (lead); project administration (lead); writing original draft (lead); writing review and editing (lead). Noga* Arwas completed conceptualization (equal); investigation (equal); writing original draft (equal); writing review and editing (lead). Michal Maimon and Micha Aviram completed methodology (equal); supervision (equal); writing review and editing (equal). Romi Bari completed data curation (lead); formal analysis (lead); methodology (equal); writing review and editing (equal). Dvir Gatt completed investigation (equal); methodology (equal); writing review and editing (equal).

\section{DATA AVAILABILITY STATEMENT}

The data that support the findings of this study are available from the corresponding author upon reasonable request.

\section{ORCID}

Inbal Golan-Tripto (1) https://orcid.org/0000-0001-6259-405X

Noga Arwas (10) https://orcid.org/0000-0003-2782-642X

Dvir Gatt (D) https://orcid.org/0000-0001-8666-5115

Aviv Goldbart (1D http://orcid.org/0000-0002-7063-4653 


\section{REFERENCES}

1. Viner RM, Russell SJ, Croker H, et al. School closure and management practices during coronavirus outbreaks including COVID-19: a rapid systematic review. Lancet Child Adolesc Health. 2020;4(5):397-404.

2. Bin Nafisah S, Alamery AH, Al Nafesa A, Aleid B, Brazanji NA. School closure during novel influenza: a systematic review. J Infect Public Health. 2018;11:657-661.

3. Rashidashid H, Ridda I, King C, et al. Evidence compendium and advice on social distancing and other related measures for response to an influenza pandemic. Paediatr Respir Rev. 2015;16:119-126.

4. Cowling BJ, Ali ST, Ng TWY, et al. Impact assessment of nonpharmaceutical interventions against COVID-19 and influenza in Hong Kong: an observational study. medRxiv. 2020:16.

5. Shen K, Yang Y, Wang T, et al. Diagnosis, treatment, and prevention of 2019 novel coronavirus infection in children: experts consensus statement. World J Pediatr. 2020:7.

6. Qin X, Zahran HS, Malilay J. Asthma-related emergency department (ED) visits and post-ED visit hospital and critical care admissions, National Hospital Ambulatory Medical Care Survey, 2010-2015. J Asthma. 2020;23:1-8.

7. Fu LS, Tsai MC. Asthma exacerbation in children: a practical review. Pediatr Neonatol. 2014;55(2):83-91.

8. Cohen HA, Blau H, Hoshen M, Batat E, Balicer RD. Seasonality of asthma: a retrospective population study. Pediatrics. 2014;133 (no. 4):e923-e932.

9. Dondi A, Calamelli E, Piccinno V, et al. Acute asthma in the pediatric emergency department: infections are the main triggers of exacerbations. BioMed Res Int. 2017;2017:9687061.

10. Castro-Rodriguez JA, Forno E. Asthma and COVID-19 in children: a systematic review and call for data. Pediatr Pulmonol. 2020;55(9): 2412-2418.

11. Lindsley AW, Schwartz JT, Rothenberg ME. Eosinophil responses during COVID-19 infections and coronavirus vaccination. J Allergy Clin Immunol. 2020;146(1):1-7.

12. Jesenak M, Brndiarova M, Urbancikova I, et al. Immune parameters and COVID-19 infection-associations with clinical severity and disease prognosis. Front Cell Infect Microbiol. 2020;10:364.

13. Kenyon CC, Hill DA, Henrickson SE, Bryant-Stephens TC, Zorc JJ. Initial effects of the COVID-19 pandemic on pediatric asthma emergency department utilization. J Allergy Clin Immunol Pract. 2020;8(8):2774-2776.

14. Allon R, Feldman O, Karminsky A, Steinberg C, Leiba R, Shavit I. Validity of the pediatric Canadian Triage acuity scale in a tertiary children's hospital in Israel. Eur J Emerg Med. 2018;25:270-273.

15. Almqvist C, Worm M, Leynaert B, Working group of GA2LEN WP 2.5 Gender. Impact of gender on asthma in childhood and adolescence: a GA2LEN review. Allergy. 2008;Jan 63(1):47-57.

16. Horowitz I, Wolach B, Eliakim A, Berger I, Gilboa S. Children with asthma in the emergency department: spectrum of disease, variation with ethnicity, and approach to treatment. Pediatr Emerg Care. 1995;11(4):240-242.

17. Amirav I, Newhouse MT. COVID-19: time to embrace MDI+ valvedholding chambers! J Allergy Clin Immunol. 2020;146(2):331.

18. Tang JW, Li Y, Eames I, Chan PKS, Ridgway GL. Factors involved in the aerosol transmission of infection and control of ventilation in healthcare premises. J Hosp Infect. 2006;64:100-114.

19. Boserup B, McKenney M, Elkbuli A. The impact of the COVID-19 pandemic on emergency department visits and patient safety in the United States. Am J Emerg Med. 2020;38(9):1732-1736.

20. Jeffery MM, D'Onofrio G, Paek H, et al. Trends in emergency department visits and hospital admissions in health care systems in 5 states in the first months of the COVID-19 pandemic in the US. JAMA Intern Med. 2020;180(10):1328-1333.
21. Ahmed T, Lodhi SH, Kapadia S, Shah GV. Community and healthcare system-related factors feeding the phenomenon of evading medical attention for time-dependent emergencies during COVID-19 crisis. BMJ Case Rep. 2020;13(8):e237817.

22. Rosenberg Danziger C, Krause I, Scheuerman O, et al. Pediatrician, watch out for corona-phobia. Eur J Pediatr. 2021;180(1):201-206.

23. Oreskovic NM, Kinane TB, Aryee E, Kuhlthau KA, Perrin JM. The unexpected risks of COVID-19 on asthma control in children. J Allergy Clin Immunol Pract. 2020;8(8):2489-2491.

24. Gupta A, Bush A, Nagakumar P. Asthma in children during the COVID-19 pandemic: lessons from lockdown and future directions for management. Lancet Respir Med. 2020;8(11):1070-1071.

25. Krivec U, Kofol Seliger A, Tursic J. COVID-19 lockdown dropped the rate of paediatric asthma admissions. Arch Dis Child. 2020;105(8): 809-810.

26. Donath $\mathrm{H}$, Zielen S, Wittekindt B, et al. Effects of the SARS-CoV2 lockdown on pediatric care in the Rhine-main area. Klin Padiatr. 2020.

27. Kuitunen I, Artama M, Mäkelä L, Backman K, Heiskanen-Kosma T, Renko M. Effect of social distancing due to the COVID-19 pandemic on the incidence of viral respiratory tract infections in children in Finland during early 2020. Pediatr Infect Dis J. 2020;39:423.

28. Hatoun J, Correa ET, Donahue SMA, Vernacchio L. Social distancing for COVID-19 and diagnoses of other infectious diseases in Children. Pediatrics. 2020;146(3):e2020006460.

29. lerodiakonouerodiakonou D, Zanobetti A, Coull BA, et al. Ambient air pollution, lung function, and airway responsiveness in asthmatic children. J Allergy Clin Immunol. 2016;137:390-399.

30. Shukla N, Sharma GK, Baruah P, Shukla VK, Gargava P. Impact of shutdown due to COVID-19 pandemic on aerosol characteristics in Kanpur, India. J Health Pollut. 2020;10(28):201201.

31. Francisco R, Pedro M, Delvecchio E, et al. Psychological symptoms and behavioral changes in children and adolescents during the early phase of COVID-19 quarantine in three European countries. Front Psychiatry. 2020;11:570164.

32. Schmidt SCE, Anedda B, Burchartz A, et al. Physical activity and screen time of children and adolescents before and during the COVID-19 lockdown in Germany: a natural experiment. Sci Rep. 2020;10(1):21780.

33. Sá CDSC, Pombo A, Luz C, Rodrigues LP, Cordovil R. COVID-19 social isolation in Brazil: effects on the physical activity routine of families with children. Rev Paul Pediatr. 2020;39:e2020159.

34. López-Bueno R, López-Sánchez GF, Casajús JA, Calatayud J, Tully MA, Smith L. Potential health-related behaviors for pre-school and school-aged children during COVID-19 lockdown: a narrative review. Prev Med. 2020;143:106349.

35. Kaye L, Theye B, Smeenk I, Gondalia R, Barrett MA, Stempel DA Changes in medication adherence among patients with asthma and COPD during the COVID-19 pandemic. J Allergy Clin Immunol Pract. 2020;8:3-2385.

36. Nascimento MS, Baggio DM, Fascina LP, do Prado C. Impact of social isolation due to COVID-19 on the seasonality of pediatric respiratory diseases. PLOS One. 2020;15(12):e0243694.

How to cite this article: Golan-Tripto I, Arwas N, Maimon MS, et al. The effect of the COVID-19 lockdown on children with asthma-related symptoms: A tertiary care center experience. Pediatric Pulmonology. 2021;56:2825-2832. https://doi.org/10.1002/ppul.25505 